\title{
EASR: An Energy Aware Source Routing with Disjoint Multipath Selection for Energy-Efficient Multihop Wireless Ad Hoc Networks*
}

\author{
Do-Youn Hwang, Eui-Hyeok Kwon, and Jae-Sung Lim \\ Graduate School of Information and Communication, Ajou University, South Korea \\ \{soyosoyo, k31001, jaslim\}@ajou.ac.kr
}

\begin{abstract}
Wireless ad hoc networks usually consist of mobile battery operated computing devices that communicate over the wireless medium. These devices need to be energy conserving so that the battery life is maximized. The energy for transmission of a packet in the wireless channel remains quite significant and may turn out to be the highest energy consuming component of the device. So, an energy-efficient communication protocol can minimize maintenance and maximize system performance. We propose an Energy Aware Source Routing (EASR) which can be efficient from network long-term connectivity point of view. In this algorithm, multiple routing paths are selected. However, only one path will be used for data transmission at a certain time among multiple paths and each path has probability to be selected. In EASR, the routing paths will be discovered without overlapped. In addition, each path hardly overhears other data transmission. We define an overhearing ratio in order to reduce the overhearing energy waste among each selected path. And we show how establish energy efficient multiple paths by making use of overhearing ratio. Our simulation results show that our proposed scheme can achieve magnitude improvement of network lifetime and reasonable packet latency time.
\end{abstract}

Keywords: EASR, energy-efficient, overhearing ratio, multipath.

\section{Introduction}

In wireless ad hoc networks, the battery of the network devices may not be replenished. So, energy-efficient communication protocol is the most important key to prolong network lifetime. Almost all of conventional routing protocols in wireless ad hoc networks find only a single optimal path and use it for every communication. It reduces the delay of data communication. However, any single path is apt to be disconnected by energy depletion. If a communication route breaks off, then we have to discover another route to maintain data transmission from source to destination. This phenomenon brings about more number of trials finding another route. As a result, we hardly prolong the networks connectivity.

The Energy Aware Routing (EAR) is one of the best known protocol selecting suboptimal paths according to the probability calculated by energy metric [1]. Energy

* This work was supported by the Korea Research Foundation Grant (KRF-2004-013-D00028). 
depletion can be prevented. However, it has another problem that the packet latency time can be increased because in EAR network nodes know sub-optimal single hop path to distribute traffic load. Therefore, we use multiple paths those are source-todestination paths in this paper. This is called Energy Aware Source Routing (EASR) protocol.

We tried to find multiple paths using the route discovery scheme of DSR [2], but almost all of the found paths are overlapped because all of the duplicated RREQs are dropped by intermediate node. So, we need another way to find maximally disjoint paths. We can use the route discovery procedure of Split Multipath Routing (SMR) which introduces the different way of route discovery instead of dropping every duplicate RREQs [3]. The intermediate nodes forward the duplicate packets which traversed through a different incoming link. By this way we can establish disjoint multipath from source to destination. However, although they are not overlapped, the overhearing effect among paths can be occurred. It causes energy waste of each selected path. Figure 1 shows the overhearing effect among nodes on each selected path. In the figure, $\{\mathrm{s}, \mathrm{j}, \mathrm{i}, \mathrm{h}, \mathrm{g}, \mathrm{f}, \mathrm{d}\}$ is a set of nodes that belong to the path 1 between nodes $s$ and $d$ and data is transmitted on it. Assume that other paths 2 and 3 are set up by $\{\mathrm{s}, \mathrm{l}, \mathrm{m}, \mathrm{n}, \mathrm{o}, \mathrm{p}, \mathrm{q}, \mathrm{d}\}$ and $\{\mathrm{s}, \mathrm{a}, \mathrm{b}, \mathrm{c}, \mathrm{k}, \mathrm{e}, \mathrm{d}\}$. Some nodes $\{\mathrm{l}, \mathrm{m}, \mathrm{n}, \mathrm{p}, \mathrm{q}, \mathrm{c}, \mathrm{k}, \mathrm{e}\}$ can overhear from the path 1 communication, then the energy of the node is wasted due to overhearing. The multiple paths communication has still the unnecessary energy waste problem.

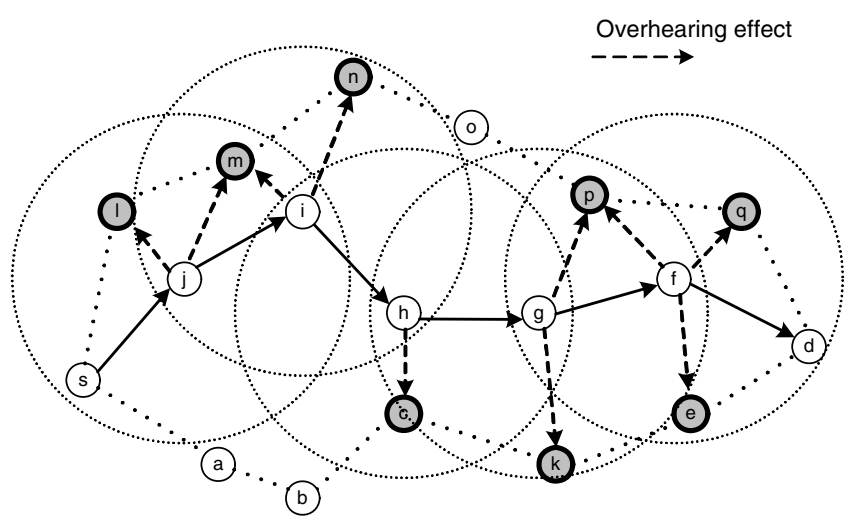

Fig. 1. Overhearing effect between two paths

Consequently, we present an energy aware source routing with a disjoint multipath selection scheme. This protocol means that data communication would use different paths at different time, and not only those paths are not overlapped but also the overhearing effect can't be occurred among the paths. We design a simple route discovery procedure to solve overhearing problem. And we define an overhearing ratio to find out the level of energy waste from overhearing effect between the paths. The source can select multiple routes by measuring overhearing ratio of each path. If the overhearing ratio is too high, the path will not be selected by the source. It can increase 
the network lifetime. In addition, providing multiple paths is profitable in wireless ad hoc network where routes are failed frequently.

The remainder of this paper is organized as follows. Section 2 describes the related works. And we introduce our EASR protocol in Section 3 in detail. Simulation results are presented in Section 4 and concluding remarks are made in Section 5.

\section{Related Works}

Finding an optimal single path is a normal approach of routing protocol for wireless ad hoc networks but it needs to be changed to prolong network connectivity.

Dynamic Source Routing (DSR) is an on-demand routing protocol for wireless ad hoc networks [2]. If any nodes want to send data packet, they flood a route request (RREQ) into the network. Any node that has a path to the destination can reply with the route reply (RREP) to the source. RREP packet contains the entire path, so the data packet can be sent properly. Almost all of the ad hoc routing protocols always use a single optimal path. Even though they use a single energy efficient path, which can deplete node energy locally and so the network is easy to be disconnected. Consequently, the source needs to flood RREQ into the network to discover another path again.

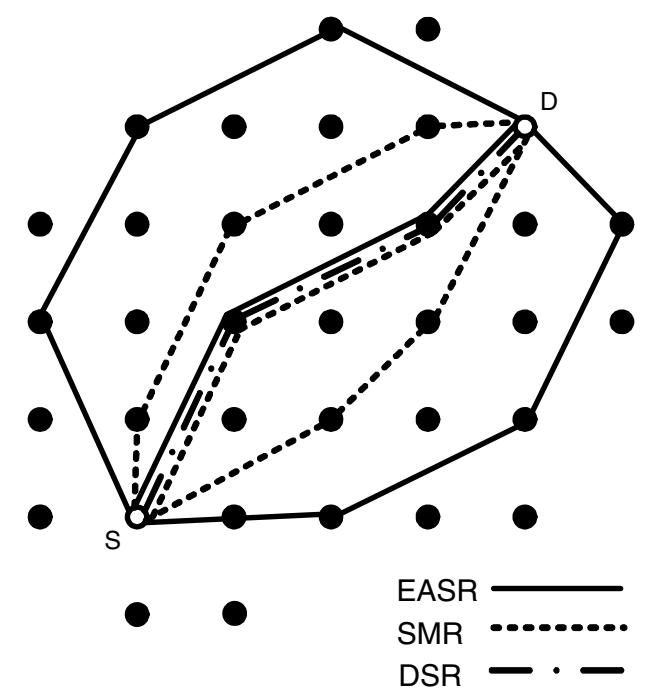

Fig. 2. Results of the route discovery of the various protocols

Split Multipath Routing (SMR) was proposed for wireless ad hoc networks [3]. The route discovery scheme of SMR is the best way to find maximally disjoint paths. It uses the source routing protocol approach where the information of the nodes that consist of the route is included in the RREQ packet. In SMR, instead of dropping every duplicate RREQ, the intermediate nodes forward the duplicate packets, if they 
are traversed through a different incoming link. And the hop count of new arriving RREQ is not larger than that of the first received RREQ. However, although they are not overlapped, we have observed in our experiments that the overhearing effect among paths is occurred. Figure 2 shows the results of the route discovery procedure of the various protocols.

\section{Proposed EASR Protocol}

\subsection{Route Discovery}

The Energy Aware Source Routing (EASR) is an on-demand routing protocol that builds multiple paths using request/reply cycles. In our algorithm, the source can select multiple disjoint paths so that the overhearing effect among paths would not be occurred. The route discovery procedure of EASR is similar to that of SMR. Disjoint multiple paths can be maintained from source to destination. However, SMR selects the paths which are just disjoint only, while EASR selects the paths which can avoid overhearing effect among them using the neighbor information that is gathered from the route reply (RREP). Whenever the source transmits data to the destination, the source chooses one of the good paths according to probabilistic fashion to prevent energy depletion.

Route Request. Our scheme has to discover maximally disjoint paths in order to avoid unnecessary energy consumption of nodes on the paths due to overhearing effect among paths. So we use the same procedure of the route request of SMR. In SMR, instead of dropping every duplicate RREQs, the intermediate nodes forward the duplicate packets that traversed through a different incoming link. And the new arriving RREQ has to also satisfy the condition that the hop count of the duplicated packets is not larger than that of the received RREQ. It is worth to note that the route request of SMR is one of the best ways to find maximally disjoint paths in spite of the increase of the number of RREQ.

Route Reply. In our protocol, we assume that all of the nodes collect neighbor node IDs. In the on-demand routing protocol, the node IDs on the entire path are recorded in the RREP, and hence the intermediate nodes can forward the RREP using this information. In addition, EASR requires additional information for establishing energy efficient multiple paths. The protocol has two phases:

a) The residual energy of all intermediate nodes is recorded in the RREP.

b) The neighbor list of each node is recorded in the RREP.

The neighbor list of each node is necessary to select good paths in the following route selection procedure. And the sum of residual energy of each path is gathered at the source node. A path selection probability of each path for the data transmission procedure will be calculated using the sum of residual energy of each path.

Route Selection. The main goal of our scheme is selecting energy efficient multiple paths from the point of view of energy-efficiency. We want to construct at least two paths that are not overlapped. Furthermore, each path has to keep a proper distance 
from the other paths to avoid overhearing effect among paths. Thus, we need a different procedure from the conventional on-demand routing protocol. First, the source node records a path of the first received RREP at the routing cache. Second, after the source receives other RREPs, the source decides whether the path information of RREP will be selected or not. If the path of RREP is overlapped or has experience in the overhearing effect, the RREP will be dropped. Except only the first arriving RREP, all of the new arriving RREPs should be considered whether they are selected or not. The following conditions make a new RREP is dropped.

a) The path of a new arriving RREP is overlapped with other paths that are selected already.

b) The neighbor lists of a new arriving RREP are overlapped with other paths that are selected.

The first condition is absolutely necessary, so all RREPs included in this condition must be dropped in the source node. However, the second condition is flexible. We can adjust a threshold of overhearing ratio defined as

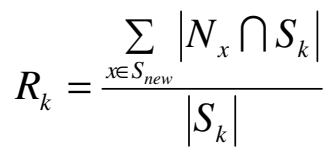

where $R_{\mathrm{k}}$ is the overhearing ratio of the $k$ th path which is already on a routing cache of the source node, in other words, the source node are already using the path for data transmission. And $N_{\mathrm{x}}$ is a neighbor list of the node $x, S_{\mathrm{k}}$ is the set of intermediate nodes which are included in the $k$ th path. Additionally, $S_{\text {new }}$ is the set of intermediate nodes of new arriving RREP, and $\left|S_{\mathrm{k}}\right|$ is the number of elements of set $S_{\mathrm{k}}$.

Figure 3 shows the new arriving RREP can be dropped because of the overhearing effect. In the figure, $S_{1}$ is $\{\mathrm{a}, \mathrm{b}, \mathrm{c}\}, S_{2}$ is $\{\mathrm{e}, \mathrm{f}, \mathrm{g}\}$ and $S_{\text {new }}$ is $\{1, \mathrm{k}, \mathrm{j}, \mathrm{i}, \mathrm{h}\}$. Next, $N_{\mathrm{b}}, N_{\mathrm{i}}$,

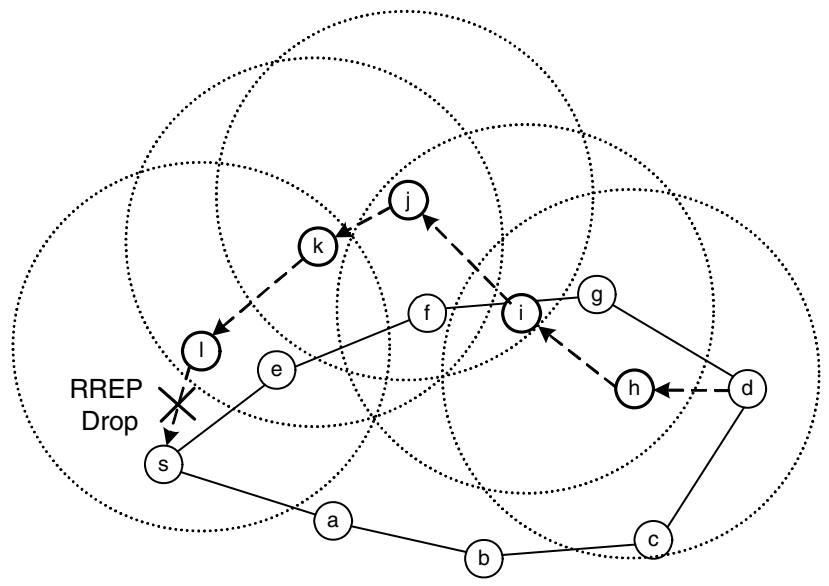

Fig. 3. An example of the EASR route selection 
$N_{\mathrm{j}}, N_{\mathrm{k}}, N_{\mathrm{l}}$ are neighbor list set of $S_{\text {new }}$, for example $N_{\mathrm{h}}$ is $\{\mathrm{i}, \mathrm{g}, \mathrm{c}\}$. By applying (1) to this case, we can acquire the value $R_{1}$ is 0.33 and $R_{2}$ is 2.33 . Then, the source node needs to decide whether to select the path of new arriving RREP or not. In the case of the figure 3, if we set the threshold of overhearing ratio to 0.4 , the new arriving RREP should be dropped. Since, the value $R_{2}$ is larger than threshold 0.4 . That is to say, accepting a new path requires that all of the overhearing ratios of each path are lower than the threshold. Because overhearing ratio indicates the extent of the energy waste due to overhearing effect of selected paths.

\subsection{Data Transmission}

The source node sends data packets to the destination with the probability of the each selected path. This means that none of the paths is used all the time to prevent energy depletion. Thus we use a similar way with EAR. But EASR is a source routing scheme, thus we can consider the number of hops from source to destination because of delay perspective. Consequently the end-to-end packet transmission delay is lower than that of EAR. Each path is assigned by the probability of path chosen according to a simple metric given by

$$
P_{n}=E_{n} / \sum_{k=1}^{S} E_{k}
$$

where $P_{\mathrm{n}}$ is the probability of $n$th path, $E_{\mathrm{n}}$ is energy metric of the nth path, and $S$ is the number of whole selected routes. This simple metric can contribute low latency with high energy efficiency. As we mentioned before, the residual energy of all intermediate nodes is recorded in the RREP, so the source node can compute probability of each path. This energy metric $E_{\mathrm{k}}$ computed as

$$
E_{k}=\left(R_{k}\right)^{\alpha} \cdot\left(1 / n_{k}\right)^{\beta}
$$

where $R_{\mathrm{k}}$ is the average residual energy of all nodes on the $k$ th path, $n_{\mathrm{k}}$ is the number of hops on the $k$ th path. The weighting factors $\alpha$ and $\beta$ can be chosen to find the energy efficient path or the path with low latency.

The energy metric is a very important component of the proposed protocol. Depending on the metric, the characteristics of our scheme can be changed substantially. We use a simple metric that can reduce delay and energy waste.

\section{Simulations and Results}

The simulations were carried out in NS-2.28 to evaluate the proposed scheme in terms of network lifetime. Simulation model is defined as a network of 50 mobile hosts placed randomly within a 1000 meter $\times 1000$ meter area, but the position of the sources and destinations is fixed in proper place in the simulation area. 5 sources and 5 destinations are selected randomly. Each source starts the data transmission at random time and it will stop after 100 seconds. However, the sources and destinations can not be neighbor of each other because in that case no routing protocol is needed. Table 1 shows the simulation parameters in detail. 
Table 1. Parameters of NS-2.28 simulators

\begin{tabular}{ll}
\hline Parameters & Value \\
\hline Packet size & 30 bytes \\
Number of nodes & 50 \\
TxPower & $30 \mathrm{~mW}$ \\
RxPower & $20 \mathrm{~mW}$ \\
Initial node energy & $30 \mathrm{Joules}$ \\
$(\alpha, \beta)$ & $(1,0.1)$ \\
Overhearing ratio threshold & $0.4 / 0.7 / 1.5$ \\
Packet arrival rate (packet/s) & $17 \sim 167$ \\
MAC protocol & IEEE $802.11 \mathrm{~b}$ \\
Simulation time & 500 seconds \\
\hline
\end{tabular}

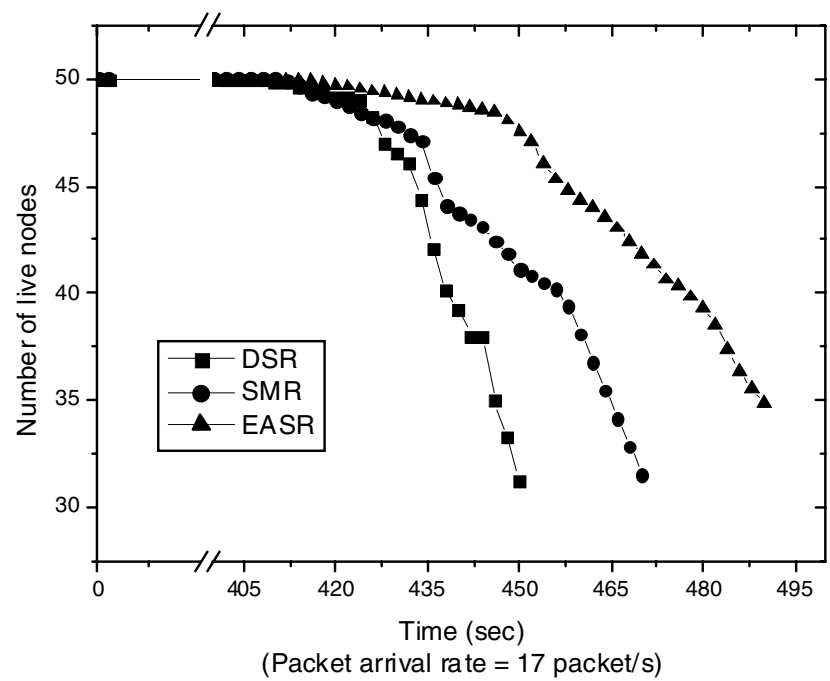

Fig. 4. Number of live nodes in networks

Figure 4 illustrates the number of live nodes with each protocol. We can see that EASR can prolong the network connectivity longer than comparable schemes such as DSR and SMR. In this simulation, we choose 0.4 for a threshold of overhearing ratio. It means that the overhearing effect is allowed only $40 \%$ of the entire path. Almost all of conventional ad hoc routing protocols such as DSR always use a single route. Even though they choose an energy efficient path, they can deplete the nodes energy locally. The path is susceptible to be disconnected. Therefore the source node of DSR needs to flood RREQ into the network to find another path again. It is observed that the number of live nodes is decreased dramatically when the route discovery procedure of DSR is used in networks. And, at 450 seconds, the source can't find any route to destination anymore. Unlike DSR, EASR discovers multiple paths and uses them for data transmission to prevent energy depletion. In addition the source node sends 
data to destination with the probability of each selected path. So we can see better performance when we compare EASR with SMR and DSR. In short, the performance of EASR is the best because the traffic load is distributed to multiple paths and each path can avoid overhearing energy waste.

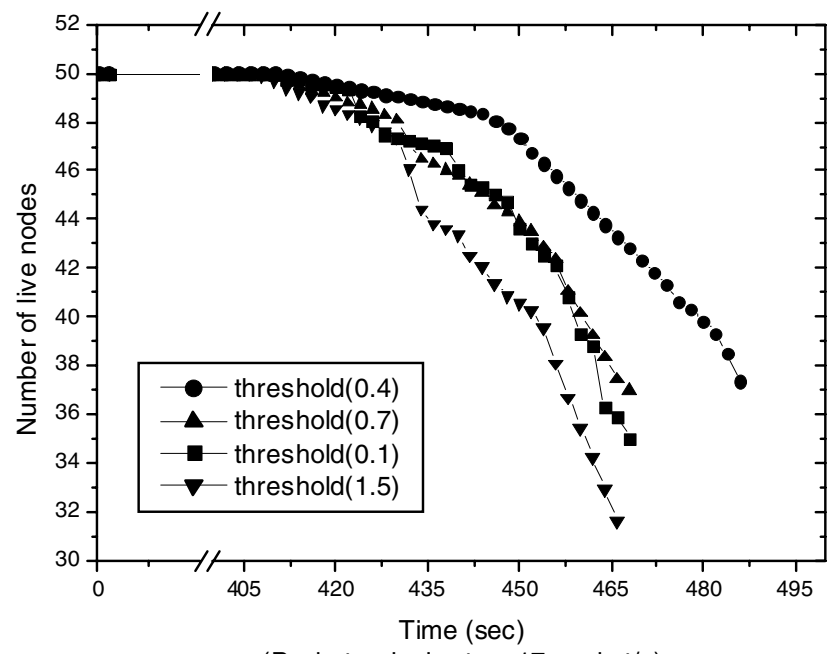

(Packet arrival rate $=17$ packet $/ \mathrm{s}$ )

Fig. 5. Number of live nodes according to threshold of overhearing ratio of EASR

Obviously, if we apply different threshold of overhearing ratio, the performance of EASR would be changed. Figure 5 shows the number of live nodes of EASR according to the threshold of overhearing ratio. The large threshold value means that most of the paths can be selected for data communication even though wasted energy due to overhearing effect is serious. Otherwise, the small threshold value means that the overhearing effect is not allowed strictly. One of the most important things is adjusting threshold level. The small threshold can achieve good performance, but if the threshold is extremely small, the source can establish only one path in most of cases. Therefore it operates like DSR. As we saw the figure 5 the performance of threshold $(0.1)$ is worse than that of threshold (0.4). In addition, if the threshold value is overly big as 1.5 , then EASR operates like SMR.

Figure 6 illustrates the end-to-end packet transmission time of each protocol. And we choose 0.4 for a threshold of overhearing ratio. Because DSR discovers a single shortest path, the performance is better than other schemes when the packet arrival rate is lower than about 80pps. However, according to increase of data rate, queuing delay is raised. The queuing delay makes the performance of each protocol worse. Especially, the end-to-end packet transmission delay of DSR is increased dramatically according to increase of data rate because DSR uses only a single shortest path for every data transmission. On the other hand, the data traffic is split into multiple routes in the case of SMR and EASR. Therefore the end-to-end packet transmission delay of SMR and EASR is increased slowly according to increase of data rate. 


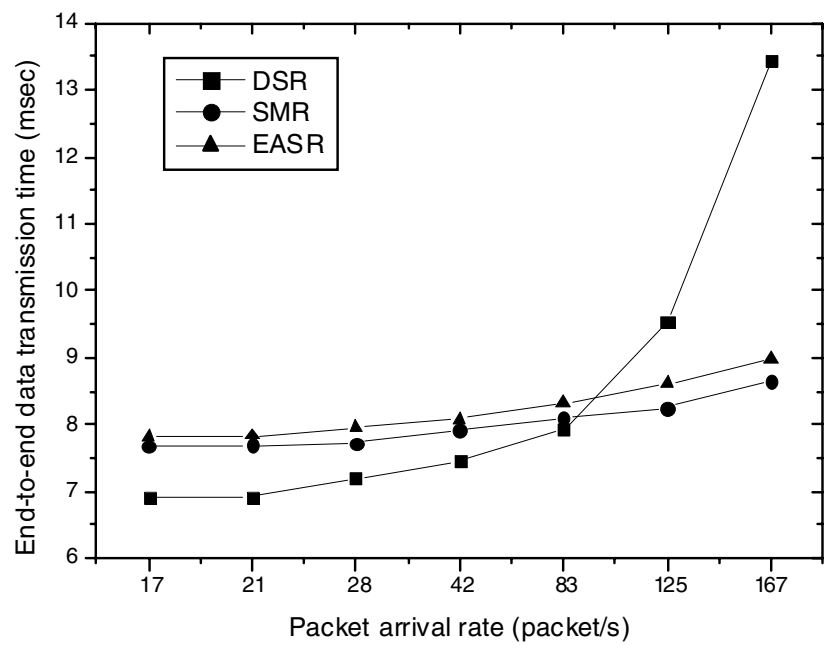

Fig. 6. End-to-end packet transmission time according to packet arrival rate

\section{Conclusions}

In this paper, we presented the Energy Aware Source Routing (EASR) protocol for energy-efficient wireless ad hoc networks and analyzed its performance through simulations. We introduced the overhearing ratio as a proper factor to control overhearing effect of each path. Additionally data propagation of EASR is performed like EAR in order to saving battery energy of ad hoc device. However, EASR is ondemand source routing protocol, therefore the delay aspect is enhanced compared with EAR obviously. Besides, maintaining multiple paths is useful in wireless networks because the source can simply use other available route without performing the route recovery process.

\section{References}

1. Rahul C. Shah and Jan M. Rabaey, "Energy Aware Routing for Low Energy Ad Hoc Sensor Networks", IEEE Wireless Communication and Networking Conference, 2002

2. D. B. Johnson and D. A. Marltz, "Dynamic Source Routing in Ad Hoc Wireless Networks," In Mobile Computing, edited by Tomasz Imielinski and Hank Korth, Chapter 5, Kluwer Academic Publishers, 1996, pp. 153-181

3. S. J. Lee and M. Gerla, "Split Multipath Routing with Maximally Disjoint Paths in Ad hoc Networks", ICC 2001.

4. C. Intanagonwiwat, R. Govindan, and D. Estrin, "Directed Diffusion: A scalable and robust communication paradigm for sensor networks", IEEE/ACM Mobicom, 2000, pp. 56-67.

5. R. Ogier, V. Rutenburg, and N. Shacham, "Distributed Algorithms for Computing Shortest Pairs of Disjoint Paths," IEEE Transactions on Information Theory, vol. 39, no. 2, Mar. 1993, pp. 443-455.

6. S. Singh, M. Woo, and C. S. Raghavendra, "Power aware routing in mobile ad hoc networks", IEEE/ACM Mobicom, Oct. 1998, pp. 181-190. 
7. J. Chang and L. Tassiulas, "Energy conserving routing in wireless ad hoc networks", IEEE Infocom, 2000, pp. 22-31.

8. Feeney, L., Nilsson, M., "Investigating the Energy Consumption of a Wireless Network Interface in an Ad Hoc Networking Environment," IEEE INFOCOM 2001

9. J. Chou, D. Pelrovis, and K. Ramchandran. "A distributed and adaptive signal processing approach to reducing energy consumption in Sensor networks:' in Proc. IEEE INFOCOM 2003, April 2003, San Francisco, CA.

10. S. Doshi, S. Bhandare, and T.X. Brown. "An on-demand minimum energy routing protocol for a wireless ad hoc network: ACM Mobile Computing and Communications Review. vol. 6. no. 3, July 2002.

11. S. Rhee, D. Seetharam and S. Liu. "Techniques for Minimizing Power Consumption in Low Data-Rate Wireless Sensor Networks" Wireless Communications and Networking Conference 2004, IEEE Vol. 3, Mar. 2004, pp. 1727 - 1731.

12. X. Li, "Energy efficient wireless sensor networks with transmission diversity," Electron. Lett., vol. 39, no. 24, pp. 1753-1755, Nov. 2003. 